\title{
Filterscape: Energy Recycling in a Creative Ecosystem
}

\author{
A. Eldridge and A. Dorin \\ Centre for Electronic Media Art, Monash University, 3800, Australia
}

\begin{abstract}
This paper extends previous work in evolutionary ecosystemic approaches to generative art. Filterscape, adopts the implicit fitness specification that is fundamental to this approach and explores the use of resource recycling as a means of generating coherent sonic diversity in a generative sound work. Filterscape agents consume and deposit energy that is manifest in the simulation as sound. Resource recycling is shown to support cooperative as well as competitive survival strategies. In the context of our simulation, these strategies are recognised by their characteristic audible signatures. The model provides a novel means to generate sonic diversity through de-centralised agent interactions.
\end{abstract}

\section{Introduction}

In recent years the ecosystem has appeared as the metaphorical cornerstone in the work of a number of electro-acoustic composers [1], performers [2] and performance theorists [3]. The unifying theme across these diverse works is that a musical performance is contingent upon a range of interacting components (social, ethnographic, musical, technological etc.) and emerges at performance time, rather than being explicitly determined in advance.

Within the field of Evolutionary Music and Art (EMA), there is a similarly burgeoning interest in ecosystemic concepts. In this field, 'ecosystem' refers to a set of systemic principles that extend the metaphors of standard Evolutionary Computation (EC): broadening the metaphor from Darwinian evolution of isolated genotypes to the digital specification of entire ecosystems may be a fruitful approach in creative contexts [4], [5]. In this case 'fitness' is no longer an explicitly pre-specified function as in standard EC, but implicit in the design of agents' interaction with their environment and one another. In parallel with broader musical trends, the resultant sonic behaviour is contingent upon the interacting components and emerges at runtime.

For the artist wishing to explore the potential of ecosystemic models, a generalisable and comprehensive body of work, similar to that which currently exists in the EC literature, has yet to be formulated. In an ongoing project, we are exploring the application of specific ecosystemic principles in audio-visual contexts (e.g. [6], [7]) with the aim of developing a conceptual and methodological tool box for EMA practitioners.

This paper addresses the issue of evolutionary 'selection pressure' and aesthetic diversity. A study in the design of a sonic ecosystem is presented in which 
energy recycling is shown to generate novel survival strategies, leading to an increase in the range of sonic behaviour that is generated.

\subsection{From Fitness Functions to Survival Strategies}

In moving from standard EC to an evolutionary ecosystemic approach the isolated genotype is embedded in an environment. An agent's chances of survival and reproduction are implicitly determined by its environmental interactions, rather than by an explicitly defined fitness function. This reflects a theoretical shift from adaptationist Darwinian evolution to extended evolution [8]. Adoption of this stance is fuelled partly by the belief that it is a more accurate reflection of the actual processes of natural evolution, and partly the intuition that this may be a more natural - and ultimately rewarding - approach to EMA.

From this ecosystemic perspective, selection pressures emerge through the evolution of agents mediated by their environment. The specification of agentenvironment interactions replaces the fitness function as the principle design challenge. This task can be informed by existing Artificial Life (Alife) models coupled with insights from ecology. For example, in many existing agent-based models, reproductive success is determined by individuals' energy acquisition ability rather than a global fitness function.

Existing models demonstrate that heterogeneity of resources in the environment can give rise to surprisingly complex agent behaviour at the population level [9]. Our intuition is that if interesting dynamics can emerge from agentenvironment interactions with fixed environments, then modelling situations in which agents can alter their environment has great potential in creative contexts, particularly in situations where coherent diversity is desirable. The application of this process of niche construction in EMA is currently under investigation.

The maintenance of diversity is central in many evolutionary music situations. Diachronic diversity can be successfully sustained through the use of coevolutionary designs that ameliorate the problem of premature fitness function convergence. In musical applications this is invariably framed as the competitive coevolution of 'singers' and 'listeners' (e.g. [10], [11]). Organism interactions are not limited to direct competition however: the myriad of symbiotic relationships observed in the natural world provide rich inspiration for achieving synchronic diversity in creative system design. The study reported here aims to increase the range of potential sonic dynamics by expanding the types of viable survival strategies. Specifically, the standard energy model deployed in agent based simulations is developed to include a simple form of energy recycling with the objective of supporting cooperative as well as competitive survival strategies.

\subsection{Energy Recycling and Cooperative Behaviour}

In models such as the early versions of Sugarscape, resources are externally supplied and agents compete to collect them by traversing a simulated landscape. Such models capture the basic flow of energy from producers to consumers in natural ecosystems. Producers are autotrophic organisms, such as green plants, 
that are able to manufacture food from the abiotic environment. Consumers are heterotrophic organisms, such as herbivores and carnivores, that rely on eating other organisms to survive. In systems such as Tierra [12] we see a slightly more complex food chain with the emergence of parasites which are secondary consumers - agents that exploit other agents to obtain energy.

The flow of energy from producers up to consumers through the trophic levels that is modeled in these systems is known as the grazer system in ecology [13]. In real-world food webs the grazer system is invariably coupled with a decomposer system. Decomposers (chiefly bacteria and fungi) act to recycle nutrients and energy from the dead organic matter produced by the organisms of the grazer system. This recycling is absolutely fundamental to ecosystem functioning but is invariably overlooked in evolutionary simulations [14]. Expanding the basic energy model to include recycling opens the door for mutualistic as well as competitive survival strategies as agents can potentially survive by reusing each other's waste as well as competing for resources.

The remainder of this paper describes the development and analysis of a sonic ecosystem that incorporates a simple form of energy recycling. The system illustrates how the design of low level interactions can give rise to a range of creatively pertinent survival strategies that include cooperative as well as competitive relations. Analysis of the system reveals how environmental structure can be manipulated to influence the prevalence of these strategies. Each survival strategy creates a sonically distinct behaviour. Increasing the repertoire of strategies therefore opens up the range of sonic possibilities.

\section{Filterscape}

Filterscape is a simple sonic ecosystem in which agents traverse a spectral filter, extracting energy from one frequency band and re-depositing it in another. Sonically, the experiment can be seen as a study in the design of a self-steering spectral filter. Aesthetically, the only constraint is that it should exhibit a coherent and varied range of behavioural dynamics. Conceptually, the study demonstrates how multiple survival strategies can emerge using simple ecosystemic principles. Technically, the aim was to investigate whether these strategies could be shaped by altering environmental structure.

\subsection{Model Details}

The filter is driven by the movement of an evolving population of agents across its frequency bands. The simulated environment is therefore a discrete, bounded 1D line. The whole population is updated asynchronously at every time step.

Energy Model. Resources are supplied externally. Uniform and non-uniform distribution regimes were compared. In the uniform case, resources are evenly distributed across the space. In the non-uniform case, the total incoming resource amount is randomly partitioned and each partition allocated to a location in the 


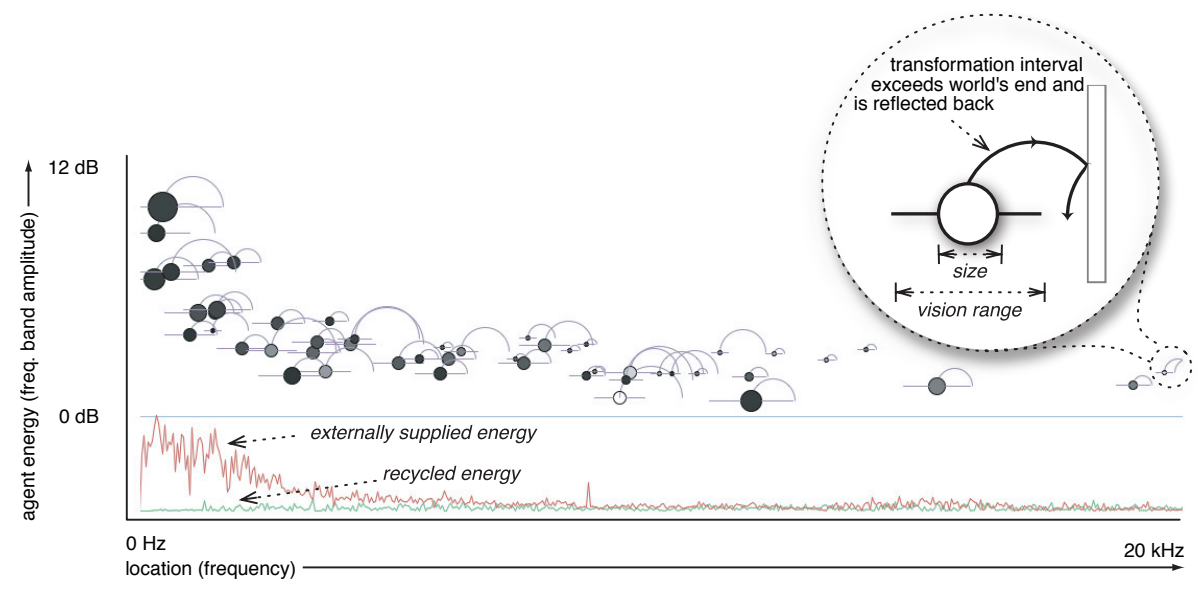

Fig. 1. Screen shot of a Filterscape visualisation. Circle diameter denotes agent size, the arc represents the transformation interval. The horizontal line through the body of each agents signifies its vision range. This image depicts the population shortly after a population explosion. Inset shows how recycled energy is reflected at boundaries.

world using a uniform random distribution. Resources are added at the same constant rate per time step in both distribution models and unused resources accrue. Energy in each location is equally available to all agents at that location. Rate of incoming energy is set to 35 units per time step.

Agent Specification. Agents are genetically specified by their size and transformation interval. An agent's size determines its vision range and metabolism, creating a trade off between energetic requirements and resource acquisition capabilities. The transformation interval determines the location of recycled energy relative to the agent's position (magnitude and direction). Each agent keeps track of its current state as described by its energy level and position. At the start of a simulation run, agents are initialised with $E=100$ initial energy units and randomly assigned size and transformation intervals.

Agent Behaviour. Agents survive by navigating to and consuming resources. Resources are transformed directly into agent energy units. At each time step, each agent moves to the location with the highest resource level within its vision range. The agent acquires half the resources at its new location. Multiple agents can occupy the same location. An agent dies if its energy level falls to zero.

Movement has an energetic cost proportional to distance moved. Irrespective of movement, a metabolic living cost is imposed as a function of size. At each time step, a percentage of this waste energy is reintroduced into the environment at a location specified by the agent's genetically determined transformation interval. 
Recycled energy is not differentiated from the external energy supply and can be used in exactly the same way. When transformations would exceed the bounds of the world, energy is reflected back (see Figure 1, inset). Metabolic living cost is set at 1 unit per unit size; $95 \%$ of metabolised energy is recycled.

Reproduction. Haploid asexual reproduction occurs for any agent when its energy level exceeds its initial endowment $(E)$ by a fixed percentage. The offspring produced acquires half the parent's energy. Size and interval are creep mutated with a fixed probability, using values drawn from a Gaussian distribution and scaled over the range of allowable values. The offspring is placed in the nearest empty location. A carrying capacity was externally imposed to prevent population explosions in this simple model, meaning that population fluctations can occur up to a given limit. Reproduction threshold, thresh $r=0.5 \times E$; $P($ mutation $)=0.1 ;$ carrying capacity $=40$.

Simulation Parameter Values. Parameter values were set experimentally such that a balance between saturation (agents do not need to move) and starvation (agents are unable to exist) was achieved, whilst maintaining a modicum of biological plausibility.

Sonic Interpretation. The combined energy levels of all agents at a particular location specify the amplitude of the corresponding frequency band. Sound is produced by convolving the array of energy values at each location with white noise and performing an inverse Fast Fourier Transform. Agents therefore act to shift energy from the spectral bin where they are located either up or down the frequency spectrum according to their genetic makeup.

\section{$3 \quad$ Results}

\subsection{Behavioural Strategies and Sonic Dynamics of the Basic Model}

With energy recycling turned on, at least three distinct strategies for resource acquisition can be observed, each generating characteristic sonic dynamics. Firstly, agents can glide slowly across the space. Secondly in this bounded world agents can loiter at an edge and reflect the energy that they recycle into locations near or within their vision range. Finally agents can exploit each other's recycling behaviour and cluster together in mutualistic groups, each taking up the waste deposited by other agents (see Figure 2).

- Gliding produces continuous wide frequency sweeps as agents move up and down the filter. This is only a viable strategy in relatively empty worlds where the resource supply is effectively continuous in space. In general they move in the direction specified by their transformation interval collecting their recently recycled energy. 


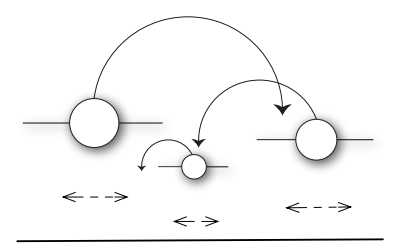

CLUSTER

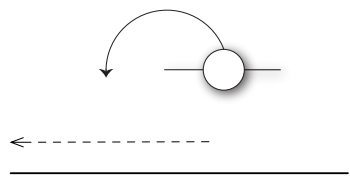

GLIDE

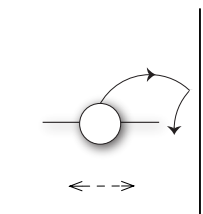

LOITER

Fig. 2. Agent formations and movement patterns for the principle survival strategies

- Loitering creates a quiet drone followed by either silence, or a large sweep across frequencies. This is an efficient strategy as agents can reflect energy back within their vision range, minimising the cost of movement. Loiterers make small movements in either direction whilst remaining close to an edge. Often loiterers are the last remaining agents in a population and are invariably precursive to a population extinction or explosion (see below).

- Clustering creates spectral clusters that drift through frequency space. Clusters can only form when a number of agents' transformation intervals are matched. When this does occur, it offers an efficient strategy as agents' movement costs are minimised.

To confirm that the observed strategies were a product of recycling rather than an artefact of some other aspect of the simulation, recycling was turned off and the external energy rate adjusted. In the absence of recycling, neither clustering nor loitering are observed, leaving gliding as the only viable survival strategy. Recycling supports the viability of survival strategies that increase the repertoire of the system as a sound work. The observation that clustering and loitering are unstable and require additional externally supplied energy led to the hypothesis that their viability is a function of resource distribution.

\subsection{Effect of Resource Distribution on Survival Strategy and Population Dynamics}

To examine whether the prevalence of the different behavioural strategies (glide, loiter, cluster) were influenced by resource distribution, the simulation was run using uniform and non-uniform resource allocation (see section 2.1).

Figure 3 shows typical plots of resource deposits made by agents under uniform (top) and non-uniform (bottom) distribution regimes. Each agent deposits energy at every timestep, therefore these give an imprint of the movement of the population in space-time. Figure 3 (top) illustrates the typical behaviour of a population with uniformly distributed resources: individuals glide smoothly back and forth through space. In contrast Figure 3 (bottom) shows a space-time plot for the loitering behaviour that is most prevalent in the non-uniform condition. A small number of loiterers hug the edge of the space from iteration 100 until 


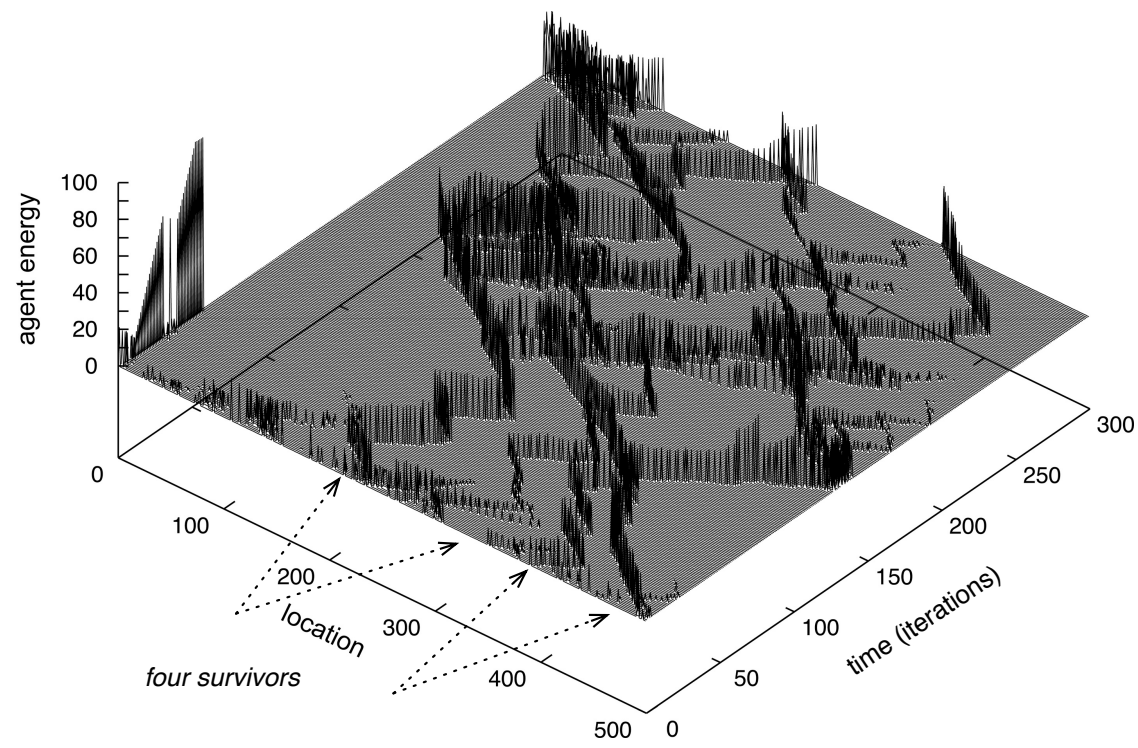

NON-UNIFORM RESOURCE DISTRIBUTION

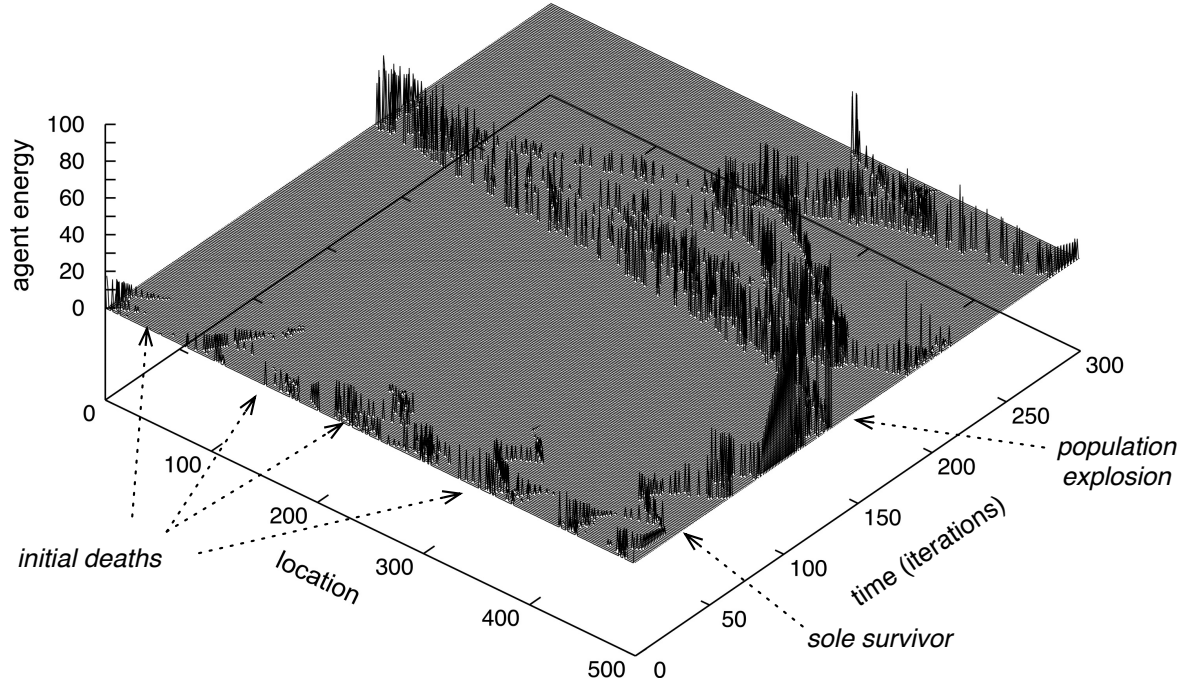

Fig. 3. Plots of energy deposits in space across 300 iterations for typical runs under uniform distribution of resources (top) and non-uniform distribution (bottom). 
around 170 when a population explosion occurs. The whole population traverses the space, creating a high to low frequency sweep. Clustering occurs under both regimes, relying primarily on the chance union of compatible agents.
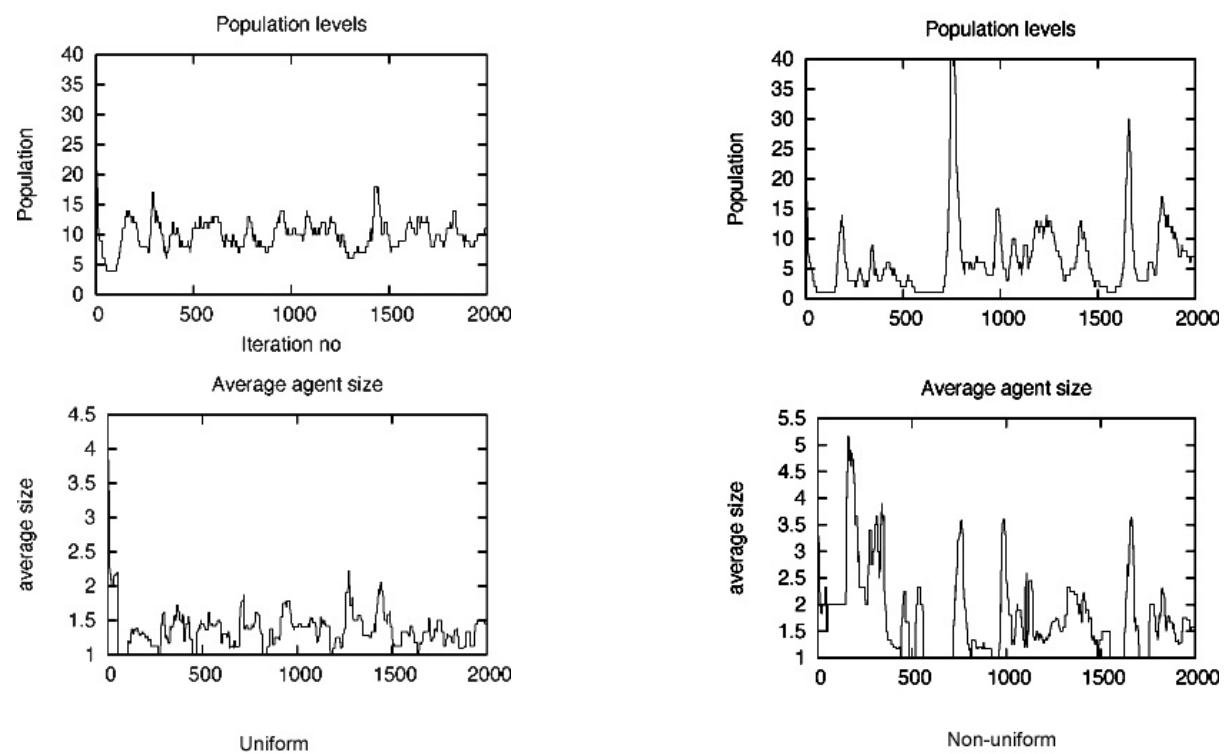

Fig. 4. Plots of population levels (top) and average agent size (bottom) for typical runs of uniformly (left) and non-uniformly (right) distributed resources.

These behavioural strategies give rise to distinct population dynamics. Loitering behaviour causes large fluctuations in population size (see Figure 4, top, right). As loiterers are often the sole survivors of a population and remain close to an edge, resources accrue in the unoccupied space. If the loiterers reproduce and create offspring that are capable of accessing adjacent resources, the resource-rich locations are rapidly harvested, creating a population explosion and migration. The time between these explosions and their size are positively correlated: the longer the world is empty the greater the amount of resources accrue and so the larger the population explosion that occurs. In contrast, when resources are supplied uniformly to create a homogenous environment, gliding behaviour predominates, and with it a more stable population is observed (Figure 4, top, left). Sonically, these variations in population size and agent position create drones and sweeps across frequency and amplitude and are far more interesting than the static fields created when only gliding behaviour is present.

Population explosions also cause transient changes in population size distribution: statistically as the population grows, size variation increases. The effect is short-lived however as recycling is less than 100\% efficient. As shown in Figure 
4 (bottom) population explosions are invariably followed by transient increases in average agent size. This effect is more pronounced when resources are distributed non-uniformly (Figure 4, bottom, right). As larger agents contain more energy their presence amplifies the effect of increased population size, creating larger variations in the overall loudness of the filter.

\section{Discussion}

As expected, the implementation of resource recycling supports symbiotic as well as competitive survival strategies which in this context increases the range of sonic behaviour of the system. In the absence of recycling, spatially isolated agents are the most competitive. Gliding behaviour dominates, creating unstructured spectral movement. The introduction of spatial recycling means that agents can also acquire energy by occupying the frequency band at which another agent deposits its energy. In the natural world this form of symbiosis is described as commensalism, referring to a relationship where one organism gains an advantage at no serious cost to the other. This is exemplified by carcass eaters who follow hunting animals, moving in to eat the hunters' leftovers.

Although explicable with hindsight, the emergence of loitering was unexpected at design time, but arises from the interaction of recycling with the bounded world. Sonically the variation of loitering, and clustering is much more interesting than the default gliding behaviour. In this simulation greater diversity is observed under a non-uniform, rather than uniform resource allocation procedure. Not only does this promote loitering, but the heterogenous structure created seems to amplify variation at the population level, further enhancing the sonic dynamics generated.

\subsection{Future Work}

In the current model, resources are undifferentiated: any agent can access any resource at any location that it occupies. Similarly, incoming and recycled resources are nutritionally equivalent. This was sufficient to demonstrate the emergence of a variety of survival strategies. The next obvious step is to model different resource types and distinguish agents according to their preference or efficiency for processing a particular type of resource. Sonically this would mean constraining agent's nutritional requirements to specific frequency bands, or samples with distinct spectro-morphological signatures. We hope that this would build a more realistic food web and increase the sonic potential of the model from the present study to a fully fledged sound work.

\section{Conclusion}

Filterscape illustrates how energy recycling can increase synchronic diversity in a sonic ecosystem: in this case by creating an sonic environment that supports 
mutualistic (clustering) as well as competitive survival strategies. Experimental manipulation of resource allocation demonstrates how the appearance of these strategies can be selectively influenced by altering basic structural properties of the world. These results support the hypothesis that heterogenous environments can support a wider range of dynamics than homogenous spaces. The ecosystemic approach is formative, but on-going experiments suggest this to be a fertile route for those interested in the emergent or collaborative potential of evolutionary art.

Acknowledgements This research is supported by Australian Research Council Discovery Project grant DP0772667. Thanks to Jon McCormack for valuable discussions and comments on early drafts.

\section{References}

1. A. DiScipio. Sound is the interface: From interactive to ecosystemic signal processing. Organised Sound, 8(3):269-277, 2003.

2. J. Bowers. Improvising machines: Ethnographically informed design for improvised electro-acoustic music. ARiADATexts, (4), 2002.

3. Simon Waters. Performance ecosystems: Ecological approaches to musical interaction in. In Proceedings of EMS, 2007.

4. J. McCormack. Artificial ecosystems for creative discovery. In D. et. al. Thierens, editor, GECCO, pages 301-307, 2007.

5. A. Dorin. The virtual ecosystem as generative electronic art. In G.R. Raidl, S.Cagnoni, and J. Branke, editors, EvoStar 2004, LNVS, volume 3005, pages 467 $-476,2004$.

6. A. Dorin. Artificial life, death and epidemics in evolutionary, generative electronic art. In Rothlauf et al, editor, EvoStar 2005, LNCS, volume 3630, pages 448 - 457, 2005.

7. A. Eldridge, A. Dorin, and J. McCormack. Manipulating artificial ecosystems. In M. Giacobini et al, editor, EvoStar 2008. LNCS, volume 4974, pages 392 - 401. Springer, Heidelberg, 2008.

8. K.N. Laland, F.J. Odling-Smee, and M.W. Feldman. Evolutionary consequences of niche construction and their implication for ecology. Proceedings of the National Academy of Science, 96:10242-10247, 1999.

9. J.M. Epstein. Growing Artificial Societies: social science from the bottom up. Brookings Institution, Washington, 1996.

10. G.M. Werner and P.M. Todd. Too many love songs: Sexual selection and the evolution of communication. In P. Husbands and I. Harvey, editors, Fourth European Conference on Artificial Life, pages 434-443. MIT Press/Bradford Books, Cambridge, MA, 1997.

11. P. Dahlstedt and M. G. Nordahl. Living melodies: Coevolution of sonic communication. Leonardo, 34(3):243-248, June 2001.

12. T. Ray. An evolutionary approach to synthetic biology: Zen and the art of creating life. Artificial Life, 1(1):195 - 226, 1994.

13. M. Begon, C. Townsend, and L. Harper. Ecology: From Individuals to Ecosystems. Blackwell Publishing, 4th edition edition, 2007.

14. A. Dorin and K.B. Korb. Building virtual ecosystems from artificial chemistry. In A. Costa, editor, Proceedings of the 9th ECAL, pages 103 -112. Springer-Verlag, 2007. 